\title{
Indirect monitoring of TORC1 signalling pathway reveals molecular diversity among different yeast strains
}

\author{
Eduardo I. Kessi-Pérez ${ }^{1}$ (D) | Francisco Salinas ${ }^{2,3}$ | Jennifer Molinet ${ }^{1}$ | Asier González ${ }^{4}$ | \\ Sara Muñiz ${ }^{5}$ | José M. Guillamón ${ }^{5}$ | Michael N. Hall ${ }^{4}$ | Luis F. Larrondo ${ }^{3,6}$ | \\ Claudio Martínez ${ }^{1,2}$
}

${ }^{1}$ Departamento de Ciencia y Tecnología de los Alimentos, Universidad de Santiago de Chile (USACH), Santiago, Chile

${ }^{2}$ Centro de Estudios en Ciencia y Tecnología de Alimentos (CECTA), Universidad de Santiago de Chile (USACH), Santiago, Chile

${ }^{3}$ Millennium Institute for Integrative Biology (iBio), Santiago, Chile

${ }^{4}$ Biozentrum, University of Basel, Basel, Switzerland

${ }^{5}$ Departamento de Biotecnología de los Alimentos, Instituto de Agroquímica y Tecnología de los Alimentos (CSIC), Paterna, Valencia, Spain

${ }^{6}$ Departamento de Genética Molecular y Microbiología, Facultad de Ciencias Biológicas, Pontificia Universidad Católica de Chile,

Santiago, Chile

Correspondence

Claudio Martínez, Centro de Estudios en Ciencia y Tecnología de Alimentos (CECTA), Universidad de Santiago de Chile (USACH), Santiago, Chile.

Email: claudio.martinez@usach.cl

Funding information

CSIC/i-LINK+, Grant/Award Number: 0946; Instituto Milenio iBio - Iniciativa Científica Milenio-MINECON; CONICYT/Beca Doctorado Nacional, Grant/Award Number: 21150700; CONICYT/PCI, Grant/Award Number: REDI170239; CONICYT/ FONDECYT, Grant/Award Numbers: 11170158 and 1150522; CONICYT/ FONDEQUIP, Grant/Award Number: EQM130158

\begin{abstract}
Saccharomyces cerevisiae is the main species responsible for the alcoholic fermentation in wine production. One of the main problems in this process is the deficiency of nitrogen sources in the grape must, which can lead to stuck or sluggish fermentations. Currently, yeast nitrogen consumption and metabolism are under active inquiry, with emphasis on the study of the TORC1 signalling pathway, given its central role responding to nitrogen availability and influencing growth and cell metabolism. However, the mechanism by which different nitrogen sources activates TORC1 is not completely understood. Existing methods to evaluate TORC1 activation by nitrogen sources are time-consuming, making difficult the analyses of large numbers of strains. In this work, a new indirect method for monitoring TORC1 pathway was developed on the basis of the luciferase reporter gene controlled by the promoter region of RPL26A gene, a gene known to be expressed upon TORC1 activation. The method was tested in strains representative of the clean lineages described so far in S. cerevisiae. The activation of the TORC1 pathway by a proline-to-glutamine upshift was indirectly evaluated using our system and the traditional direct methods based on immunoblot (Sch9 and Rps6 phosphorylation). Regardless of the different molecular readouts obtained with both methodologies, the general results showed a wide phenotypic variation between the representative strains analysed. Altogether, this easy-to-use assay opens the possibility to study the molecular basis for the differential TORC1 pathway activation, allowing to interrogate a larger number of strains in the context of nitrogen metabolism phenotypic differences.
\end{abstract}

\section{KEYWORDS}

microculture, natural variation, Saccharomyces cerevisiae, TORC1 pathway

\section{I INTRODUCTION}

The yeast Saccharomyces cerevisiae is a species of industrial importance given its role in the production of bread and various alcoholic beverages, being the main species responsible for the alcoholic fermentation in the process that involves the transformation of grape must into wine (Pretorius, 2000). One of the main problems in the wine industry is the deficiency of nitrogen sources in the grape must, which are the key factors regulating the biomass content during the fermentation process and directly impacting the fermentation rate (Varela, Pizarro, \& Agosin, 2004). Thus, nitrogen deficiencies can lead to stuck or sluggish fermentations, reducing the fermentation rate and generating economic losses for the industry (Taillandier, Ramon Portugal, Fuster, \& Strehaiano, 2007). 
Recently, the TORC1 signalling pathway activation during the fermentation process has gotten renewed attention due to its central role in nitrogen metabolism regulation (Tesniere, Brice, \& Blondin, 2015). TOR kinases are key components of this eukaryotic signalling pathway that connects nutrient sufficiency to growth, promoting anabolic processes such as protein synthesis and ribosome biogenesis. There are two kinases (Tor1 and Tor2) in S. cerevisiae that are part of two protein complexes (TORC1 and TORC2), of which TORC1 is inhibited by rapamycin (Loewith et al., 2002; Loewith \& Hall, 2011). Nutrients, especially nitrogen sources, activate TORC1, which lead to two main effectors of this pathway: the Sch9 kinase and the Tap42-PP2A phosphatase complex (Broach, 2012; Loewith \& Hall, 2011).

Although proximal and distal effectors of TORC1 are well characterized, the mechanism by which nitrogen sources activate TORC1 is not completely understood (Conrad et al., 2014; Gonzalez \& Hall, 2017). In this regard, an amino acid-dependent mechanism of TORC1 activation through the EGO complex (EGOC) has been determined, whose main components are the GTPases Gtr1 and Gtr2 (Hatakeyama \& De Virgilio, 2016; Powis \& De Virgilio, 2016). However, it is unknown how amino acids are sensed, with the exception of leucine, which is thought to be sensed by the leucil-tRNA synthetase (Bonfils et al., 2012). The EGOC-dependent activation of TORC1 occurs rapidly but transiently by both poor and preferred nitrogen sources. In general, glutamine, glutamate, asparagine, and ammonium sustains high specific growth rate in yeast and are considered as preferred nitrogen sources. Conversely, proline, allantoin, and urea allow slow growth rate in yeast and are considered poor or nonpreferred nitrogen sources (Crepin, Nidelet, Sanchez, Dequin, \& Camarasa, 2012). Only preferred sources promote sustained activation coupled with an accumulation of intracellular glutamine, but independently of EGOC (Stracka, Jozefczuk, Rudroff, Sauer, \& Hall, 2014). Furthermore, constitutive activity of EGOC fails to suppress the TORC1 signalling defect under ammonium deprivation (Binda et al., 2009). Thus, there is an alternative mechanism of TORC1 activation independent of EGOC in which the participating proteins have not been fully determined (Chantranupong, Wolfson, \& Sabatini, 2015; Gonzalez \& Hall, 2017), with only suggested actors such as Pib2 protein (Kim \& Cunningham, 2015; Michel et al., 2017; Tanigawa \& Maeda, 2017; Ukai et al., 2018; Varlakhanova, Mihalevic, Bernstein, \& Ford, 2017).

New methodologies to study the activation of the TORC1 pathway in response to nitrogen sources have been developed, such as the recently developed in vitro TORC1 assay based on phosphorylation of 4EBP1, a well-known target of mammalian TORC1 (Tanigawa \& Maeda, 2017). Similarly, two methods based on immunoblot detection of TORC1 targets have proved a direct survey of its activity. The first of them uses the phosphorylation of the Sch9 kinase as readout, the best characterized TORC1 direct target in yeast (Stracka et al., 2014), whereas the second uses the phosphorylation of the target ribosomal protein S6 (Rps6; Gonzalez et al., 2015; Yerlikaya et al., 2016). These methods have allowed the phenotyping of TORC1 pathway activation in response to different amino acids. However, current methods are laborious, making difficult the analysis of larger number of yeast strains.

In this scenario, an alternative to study the TORC1 pathway is the use of genetic approaches, such as those that have been used to shed light into the molecular bases that underlie the phenotypic variability in nitrogen consumption in yeasts (Brice, Sanchez, Bigey, Legras, \& Blondin, 2014; Contreras et al., 2012; Cubillos et al., 2017; Gutierrez, Beltran, Warringer, \& Guillamon, 2013; Ibstedt et al., 2015; Jara et al., 2014). However, linkage approaches require phenotyping of a larger number of strains, which is unaffordable for monitoring TORC1 activity using the abovementioned methodologies based on immunoblot detection.

In this work, a new microculture-based methodology was developed to indirectly evaluate TORC1 activation in a nitrogen upshift experiment. Our approach utilizes the luciferase reporter gene controlled by the promoter region of RPL26A gene, a gene known to be expressed upon TORC1 activation, resulting in an indirect measuring of TORC1 activation. We used our method to indirectly evaluate TORC1 activity in four yeast strains belonging to the main phylogenetic lineages described so far, showing the existence of natural variation in TORC1 signalling pathway activation in S. cerevisiae.

\section{2 | MATERIALS AND METHODS}

\section{1 | Yeast strains and plasmids}

The strains used in this work correspond to stable haploid versions of strains representative of four clean lineages previously described for S. cerevisiae (Liti et al., 2009). These strains are YPS128 (North American, "NA"), Y12 (Sake, "SA"), DBVPG6044 (West African, "WA"), and DBVPG6765 (Wine/European, "WE") (Cubillos, Louis, \& Liti, 2009). Strains were transformed using lithium acetate method (Gietz \& Schiestl, 2007) with the pJU733 plasmid, which has the SCH9-3xHA insert and URA3 gene as selectable marker (Urban et al., 2007). The pRS426 plasmid carrying the firefly luciferase reporter gene and URA3 gene as selection marker (Luc-URA3 construct) was previously described (Salinas et al., 2016). This firefly luciferase reporter gene is a destabilized version that allows real-time quantification of gene expression in vivo (Rienzo, Pascual-Ahuir, \& Proft, 2012). The construct Luc-URA3 was amplified by polymerase chain reaction and used to replace the endogenous RPL26A open reading frame (ORF). Additionally, strains carrying GTR1 deletion were generated replacing the ORF by the hygromycin cassette. All strains used are listed in Table 1.

\section{2 | Selection of RPL26A}

A revision of previously described genes responding to TORC1 pathway was carried out to select candidate genes, considering those genes whose expression is strongly activated by the TORC1 signalling pathway. From the analysis of the TORC1-dependent transcriptome (Oliveira et al., 2015), we selected the ribosomal protein encoding gene RPL26A, due to its minor effects on translation and low pleiotropic effects generated by its deletion in a laboratory genetic background, according to the Saccharomyces Genome Database (www.yeastgenome.org).

\section{3 | Indirect monitoring of the TORC1 pathway activation in microculture conditions}

The activation of the TORC1 pathway in the strains carrying the LucURA3 reporter construct under the control of the RPL26A promoter 
TABLE 1 Yeast strains used in this work

\begin{tabular}{|c|c|c|}
\hline Name & Relevant genotype & Source/reference \\
\hline YPS128 a ("NA a") & Mat a, ho::HygMX, ura3::KanMX & (Cubillos et al., 2009) \\
\hline Y12 a ("SA a") & Mat $a$, ho::HygMX, ura3::KanMX & (Cubillos et al., 2009) \\
\hline DBVPG6765 a ("WE a") & Mat $a$, ho::HygMX, ura3::KanMX & (Cubillos et al., 2009) \\
\hline YPS128 a ("NA a") & Mat $\alpha$, ho::NatMX, ura3::KanMX & (Cubillos et al., 2009) \\
\hline Y12 a ("SA a") & Mat $\alpha$, ho::NatMX, ura3::KanMX & (Cubillos et al., 2009) \\
\hline DBVPG6765 a ("WE a") & Mat $\alpha$, ho::NatMX, ura3::KanMX & (Cubillos et al., 2009) \\
\hline YCO22 & YPS128 a pJU733 & This work \\
\hline YCO23 & Y12 a pJU733 & This work \\
\hline YCO24 & DBVPG6044 a pJU733 & This work \\
\hline YCO25 & DBVPG6765 a pJU733 & This work \\
\hline YC030 & DBVPG6765 a rpl26a::Luc-URA3 & This work \\
\hline YC031 & YPS128 a rpl26a::Luc-URA3 & This work \\
\hline YC032 & Y12 a rpl26a::Luc-URA3 & This work \\
\hline YC033 & DBVPG6044 a rpl26a::Luc-URA3 & This work \\
\hline YC034 & DBVPG6765 a rpl26a::Luc-URA3 & This work \\
\hline YC043 & YC028xYC034 & This work \\
\hline YC231 & YC032 gtr1::HygMX & This work \\
\hline YC233 & YC034 gtr1::HygMX & This work \\
\hline
\end{tabular}

Note. NA: North American; SA: Sake; WA: West African; WE: Wine/European.

$\left(P_{R P L 26 A}\right)$ was evaluated by monitoring the optical density at $600 \mathrm{~nm}$ $\left(\mathrm{OD}_{600}\right)$ and luminescence of the cells in microculture conditions (Salinas et al., 2016). We used a nitrogen (proline-to-glutamine or proline-to-leucine) upshift experiment, where the strains were grown until $\mathrm{OD}_{600} \sim 0.8$ at $30^{\circ} \mathrm{C}$ in 96 -well plates containing $300 \mu \mathrm{l}$ of yeast minimal medium ( $1.7 \mathrm{~g} / \mathrm{L}$ yeast nitrogen base without amino acids and without ammonium sulphate and $20 \mathrm{~g} / \mathrm{L}$ glucose) with proline $(0.5 \mathrm{mg} /$ $\mathrm{ml}$ ) as the only nitrogen source (YMM + Pro), supplemented with luciferin (1 mM) (Figure 1). Then, $10 \mu$ of glutamine or leucine $(15 \mathrm{mg} / \mathrm{ml}$; $0.5 \mathrm{mg} / \mathrm{ml}$ final concentration) was added. Luminescence was measured up to $12 \mathrm{~h}$ using 5-min intervals in a Cytation 3 microplate reader (BioTek, USA). To check that luciferin is not limiting in the production of luminescence, we also added luciferin ( $25 \mathrm{mM}$; $1 \mathrm{mM}$ final concentration) together with the glutamine pulse (Figure S3). All the

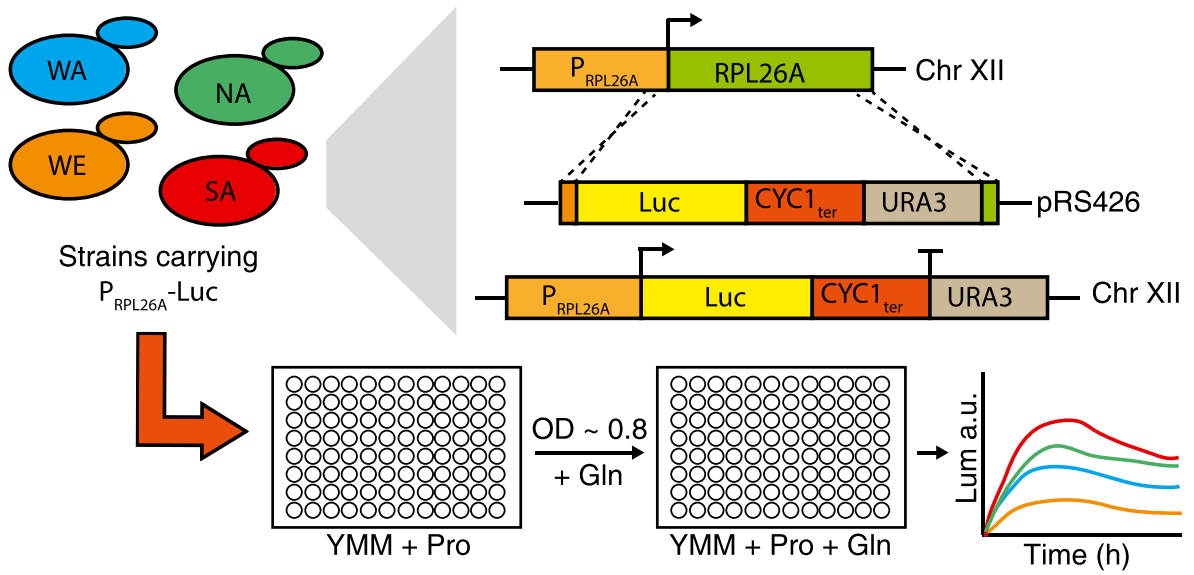

FIGURE 1 Method overview for indirect monitoring TORC1 activation in different yeast strains. The RPL26A ORF was replaced by the firefly luciferase, which is carrying the cytochrome $c$ transcriptional terminator $\left(C Y C 1_{\text {ter }}\right)$ and URA3 as selectable marker. This strategy allows luciferase expression under the endogenous RPL26A promoter $\left(P_{R P L 26 A}\right)$ control and without copy number bias. The four representative strainsYPS128 (North American, "NA"), Y12 (Sake, "SA"), DBVPG6044 (West African, "WA"), and DBVPG6765 (Wine/European, "WE")-were evaluated in a nitrogen upshift experiment, growing them in a 96-well plate with YMM plus proline (YMM + Pro) medium and adding glutamine (Gln) as nitrogen source when the strains reached $\mathrm{OD}_{600} \sim 0.8$. The luminescence (Lum) of the cells was recorded after the nitrogen pulse every 5 min. $\mathrm{OD}_{600}$ : optical density at $600 \mathrm{~nm}$ [Colour figure can be viewed at wileyonlinelibrary.com] 
microcultivation experiments were carried out in three independent biological replicas.

\subsection{Analysis of growth curves}

Relative fitness variables (growth parameters) for each strain were calculated as previously described (Kessi-Perez et al., 2016; Warringer et al., 2011). Briefly, efficiency of proliferation (population density change), rate of proliferation (population doubling time), and lag of proliferation were extracted from high-density growth curves using Gompertz growth equation (Yin, Goudriaan, Lantinga, Vos, \& Spiertz, 2003). Statistical analysis of these parameters consisted in Welch two sample $t$ tests, which were performed using R software (R-CoreTeam, 2013).

\subsection{Evaluation of the activation of TORC1 pathway by immunoblot}

The activation of theTORC1 pathway in the strains carrying the pJU733 plasmid was evaluated by assessing both the phosphorylation of its proximal effector Sch9 and the ribosomal protein Rps6 in a nitrogen (proline-to-glutamine) upshift experiment (Gonzalez et al., 2015; Stracka et al., 2014). Briefly, strains were grown in flasks containing $50 \mathrm{ml}$ of $\mathrm{YMM}+$ Pro medium until $\mathrm{OD}_{600} \sim 0.8$, and then $700 \mu \mathrm{l}$ of glutamine $(25 \mathrm{mg} / \mathrm{ml} ; 0.5 \mathrm{mg} / \mathrm{ml}$ final concentration) was added. Samples were taken at different time points $(0,5,15$, and $30 \mathrm{~min})$ to perform protein extraction and subsequent immunoblot as previously described (Gonzalez et al., 2015). To evaluate Sch9 phosphorylation, cell extracts were subjected to chemical cleavage with 2-nitro-5thiocyanatobenzoic acid (Sigma). Antibodies used included anti-HA (Cell Signaling Technology Cat\# 2367S, RRID:AB_10691311), phosphoSer235/Ser236-S6 (Cell Signaling Technology Cat\# 2211, RRID: AB_331679), RPS6 (Abcam Cat\# ab40820, RRID:AB_945319), peroxidase-monoclonal mouse antirabbit IgG (Jackson ImmunoResearch Labs Cat\# 211-032-171, RRID:AB_2339149), and peroxidase-goat antimouse IgG (Jackson ImmunoResearch Labs Cat\# 115-035-174, RRID:AB_2338512).

\section{3 | RESULTS AND DISCUSSION}

\subsection{A new indirect method for monitoring TORC1 activation}

We developed a new method to evaluate TORC1 activation by monitoring the luminescence produced by yeast cells in microculture conditions. We used the method to indirectly characterize TORC1 activity in four strains representative of clean lineages previously described in S. cerevisiae (Liti et al., 2009). We chose the strains YPS128 (NA), Y12 (SA), DBVPG6044 (WA), and DBVPG6765 (WE). These four strains were transformed with the reporter construct (Luc-URA3), designed to replace the endogenous ORF of RPL26A, such that luciferase expression is now under the control of the endogenous RPL26A promoter $\left(P_{R P L 26 A}\right)$. We selected the RPL26A promoter as readout of our system because RPL26A gene showed strong expression upon TORC1 activation (Figure S1, adapted from Oliveira et al., 2015).
The growth and luciferase expression of the strains were evaluated in $\mathrm{YMM}+$ Pro medium, monitoring both the $\mathrm{OD}_{600}$ and luminescence of the cultures over time (Figure 1). Once cells reached $\mathrm{OD}_{600} \sim 0.8$, a pulse of glutamine was added to continue recording luciferase expression until $12 \mathrm{~h}$ (Figure 1). It is important to remark that we used strains auxotroph for uracil (Cubillos et al., 2009), being the unique auxotrophy present in those strains, and therefore, the reporter construction has URA3 as selectable marker, avoiding the use of uracil in the YMM medium, which can be used as an alternative nitrogen source.

Initially, we evaluated the growth and luciferase expression under the control of $P_{R P L 26 A}$ in the four strains expressing the reporter gene in YMM + Pro medium, showing a maximum level of luciferase expression at the beginning of the exponential phase and before $\mathrm{OD}_{600} \sim 0.8$ (Figure 2). This behaviour is commonly observed for yeast promoters, with activity during a transient period at the exponential growth phase (Dolz-Edo, Rienzo, Poveda-Huertes, Pascual-Ahuir, \& Proft, 2013; Rienzo et al., 2012; Rienzo et al., 2015). Interestingly, the WA strain showed a slower growth in comparison with the other three strains in this medium, reaching $\mathrm{OD}_{600} \sim 0.8$ at later time points (Figure 2). Considering that $\mathrm{OD}_{600} \sim 0.8$ is a requisite to perform the nitrogen upshift experiment, we overcome this problem by increasing 10 -fold the concentration of WA strain at the beginning of the experiment, allowing us to evaluate the four strains in a single experiment. Additionally, we compared the growth parameters (lag time, rate, and efficiency) for the strains carrying RPL26A deletion by luciferase ( $r p / 26 a \Delta)$ versus the wild-type (WT) strains. We observed a small growth defect in the rpl26a $\Delta$ strains compared with the WT strains when grown in YMM + Pro, with statistically significant differences in lag time and growth rate for the NA genetic background (Figure 2 and Table 2). Overall, the results showed that RPL26A promoter is active at the beginning of the exponential phase and before $\mathrm{OD}_{600} \sim 0.8$, avoiding interferences with the signal produced by a nitrogen pulse at this OD. Additionally, RPL26A deletion generated minor effects over the growth parameters under the culture conditions assayed, in all four genetic backgrounds evaluated.

We then performed an upshift nitrogen experiment in a microplate reader for the four strains simultaneously, adding glutamine at $\mathrm{OD}_{600} \sim 0.8$ and monitoring the luminescence of cells during $12 \mathrm{~h}$ after glutamine addition. In all strains, the luciferase expression increased rapidly and then decreased to background levels 10-12 h after the nitrogen pulse (Figure 3a). The NA and WA strains showed the more similar behaviour, with a similar first maximum of luciferase expression at 2-3 $\mathrm{h}$ after nitrogen pulse and a second maximum at 6-8 h (Figure 3 and Table 3). The WE strain showed the smaller first maximum of luciferase expression but with a comparable second maximum with NA and WA strains, whereas SA strain showed the biggest first maximum and no second expression peak (Figure 3 and Table 3 ).

We evaluated the robustness of our method using strains carrying different mating types. We observed similar results when cells were subjected to a nitrogen upshift experiment (Figure S2). Moreover, we demonstrated that luciferin depletion over time does not affect the results obtained, which was corroborated by performing a simultaneous addition of glutamine and luciferin in the nitrogen upshift experiment (Figure S3). Finally, we assessed the capacity of our 
(a)

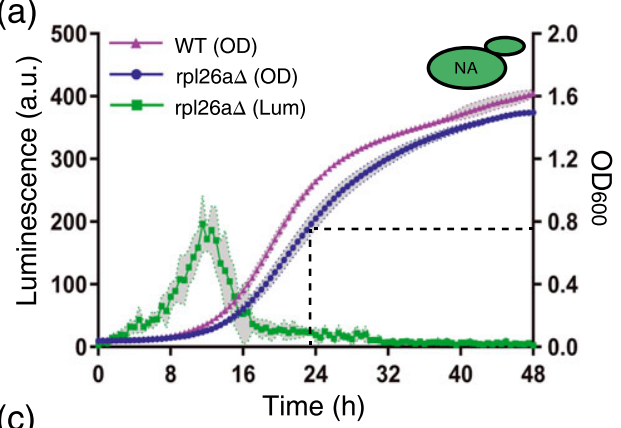

(c)

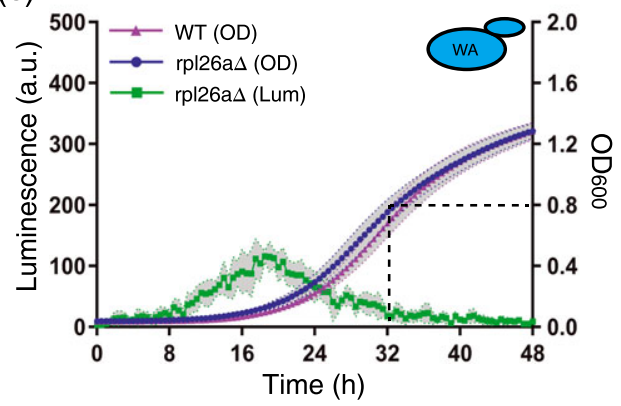

(b)

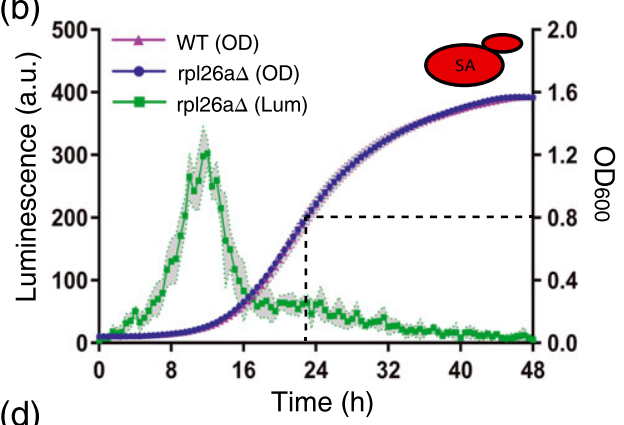

(d)

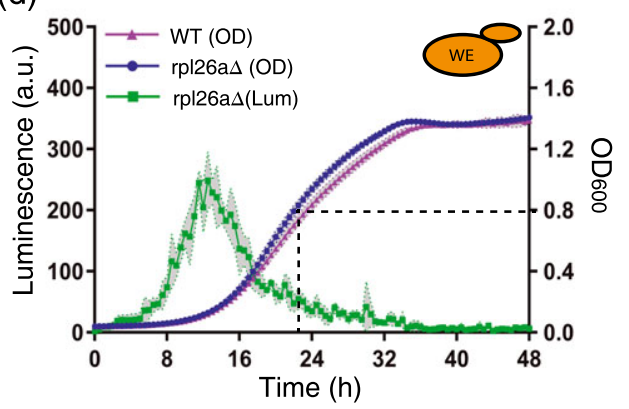

FIGURE 2 Growth and luciferase expression for strains carrying the reporter construct. The strains (a) NA, (b) SA, (c) WA, and (d) WE were transformed with the Luc-URA3 construct replacing the RPL26A ORF. These strains (rpl26a $\Delta$ ) and their wild-type (WT) versions were grown in $\mathrm{YMM}+$ Pro medium plus luciferin in microculture conditions, monitoring the $\mathrm{OD}_{600}$ and the luminescence (Lum) of the yeast cells over time. Black dashed lines show the time at which an $\mathrm{OD}_{600} \sim 0.8$ was reached by strains carrying the luciferase. Plotted values correspond to the average of three biological replicates, with their standard error represented by shadow regions (mean \pm SEM). NA: North American; SA: Sake; WA: West African; WE: Wine/European; $\mathrm{OD}_{600}$ : optical density at $600 \mathrm{~nm}$ [Colour figure can be viewed at wileyonlinelibrary.com]

TABLE 2 Growth parameters for WT strains and its version carrying RPL26A deletion

\begin{tabular}{llll} 
Strain & Efficiency $\pm S D$ & Rate $\pm S D\left(h^{-1}\right)$ & Lag $\pm S D(h)$ \\
\hline NA (WT) & $1.582 \pm 0.039$ & $0.081 \pm 0.001$ & $11.05 \pm 0.39$ \\
\hline NA $(r p / 26 a \Delta)$ & $1.542 \pm 0.016^{\text {ns }}$ & $0.070 \pm 0.005^{\mathrm{a}}$ & $12.48 \pm 0.67^{\mathrm{a}}$ \\
\hline SA (WT) & $1.607 \pm 0.011$ & $0.081 \pm 0.004$ & $13.18 \pm 0.47$ \\
\hline SA (rpl26a $\Delta)$ & $1.626 \pm 0.010^{\mathrm{a}}$ & $0.078 \pm 0.004^{\text {ns }}$ & $12.66 \pm 0.29^{\text {ns }}$ \\
WA (WT) & $1.518 \pm 0.018$ & $0.060 \pm 0.006$ & $19.95 \pm 0.59$ \\
WA (rpl26a $\Delta)$ & $1.531 \pm 0.039^{\text {ns }}$ & $0.053 \pm 0.002^{\text {ns }}$ & $18.11 \pm 1.07^{\mathrm{a}}$ \\
\hline WE (WT) & $1.440 \pm 0.038$ & $0.076 \pm 0.004$ & $12.61 \pm 0.28$ \\
WE (rpl26a $\Delta)$ & $1.425 \pm 0.007^{\text {ns }}$ & $0.087 \pm 0.002^{\mathrm{a}}$ & $12.65 \pm 0.42^{\text {ns }}$
\end{tabular}

Note. ${ }^{a}$ : $r p l 26 a \Delta$ value significantly different form WT value ( $p$ value $<0.05$ ).

${ }^{n s}$ : rpl26a $\Delta$ value not significantly different form WT value ( $p$ value $\geq 0.05$ ).

SD: standard deviation; WT: wild type; NA: North American; SA: Sake; WA: West African; WE: Wine/European.

method to be used in haploid and diploid strains. For this, we generated a hybrid strain using the phenotypically more different strains (SA and WE strains), observing in the hybrid an intermediate phenotype with respect to the parent strains in nitrogen upshift experiments (Figure S4).

In conclusion, using our method, the SA and WE strains showed the more dissimilar phenotypes for luciferase expression after a nitrogen (proline-to-glutamine) upshift experiment, which can be considered an indirect measure of the TORC1 activity. Previously, these strains were also different for other phenotypes such as fermentation kinetics and fungicide resistance (Kessi-Perez et al., 2016), nitrogen consumption (Jara et al., 2014), and oenological traits (Salinas et al.,
2012). The type of experiment performed in those studies and others, such as bulked segregant analysis (BSA) or comparative genomics, requires larger number of strains to be evaluated (Mackay, Stone, \& Ayroles, 2009). Thus, the new method here developed could be an important tool to continue unravelling genetic determinants involved in the nitrogen sensing associated with the activation of the TORC1 signalling pathway.

\subsection{Confirming phenotypic variability in TORC1 pathway activation between yeast strains}

To corroborate the phenotypic diversity seen by our method, we evaluated Sch9 phosphorylation by Western blot in a proline-to-glutamine upshift experiment as readout of TORC1 activation (Figure 4). In general, phosphorylation of Sch9 increased after 5 min of glutamine addition, decreased after $15 \mathrm{~min}$, and increased again at $30 \mathrm{~min}$, consistent with results previously described (Stracka et al., 2014). This behaviour was observed in NA, SA, and WA strains, with the SA strain showing the greater activation at 30 min (Figure 4a,b). However, the WE strain appears to lack a reactivation at $30 \mathrm{~min}$, even though it shows a great activation $5 \mathrm{~min}$ after the glutamine pulse (Figure 4a,b). In general, these results are consistent with previously described observation where glutamine can activate TORC1 both dependent and independent of EGOC, with the activation at $5 \mathrm{~min}$ being EGOC dependent and the activation at 30 min being EGOC independent (Stracka et al., 2014). Interestingly, although the WA strain showed the expected behaviour for Sch9 phosphorylation (Figure 4a,b), the unphosphorylated isoform of Sch9 was highly abundant in this strain, confirming the variability in TORC1 activity for the analysed strains. 
(a)
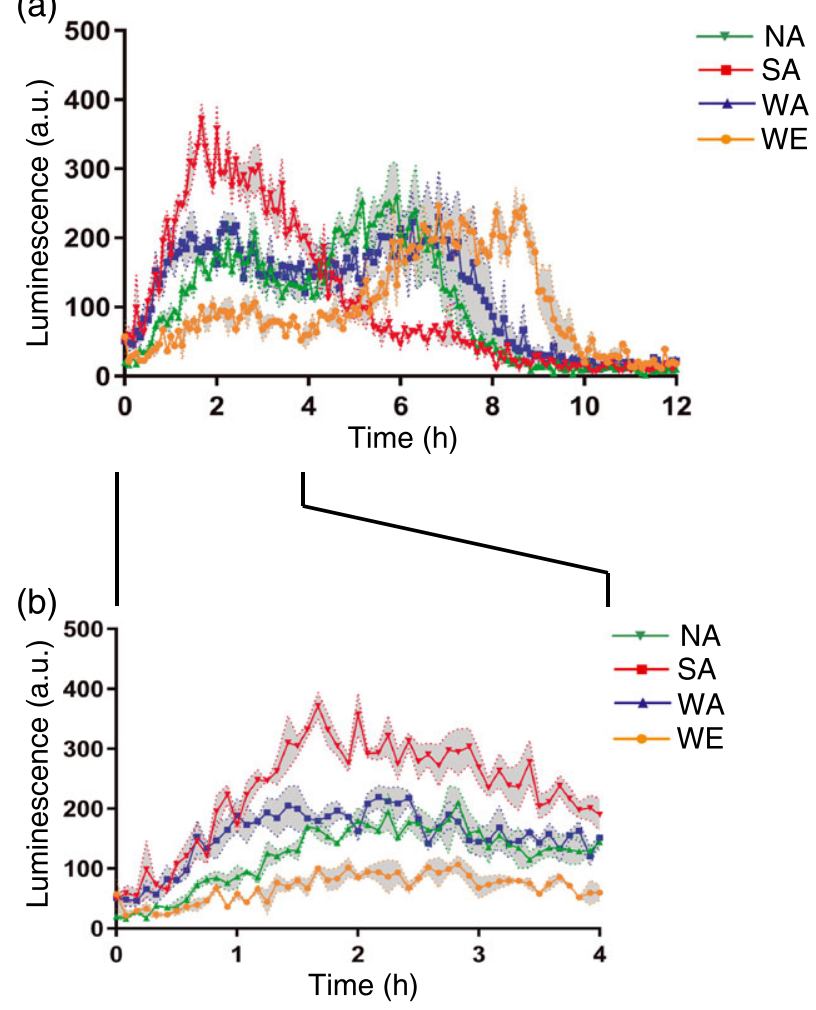

FIGURE 3 Nitrogen upshift experiment using the new method developed. Luminescence (Lum) differences between strains were evaluated in microculture conditions after a pulse of glutamine. The luminescence was recorded until $12 \mathrm{~h}$ after nitrogen pulse (a). A zoom in of the first $4 \mathrm{~h}$ after the nitrogen (proline-to-glutamine) upshift experiment (b). In both panels ( $\mathrm{a}$ and $\mathrm{b}$ ), the time $\mathrm{O} \mathrm{h}$ corresponds to the addition of glutamine. Plotted values correspond to the average of three biological replicates, with their standard error represented by shadow regions (mean \pm SEM). NA: North American; SA: Sake; WA: West African; WE: Wine/European [Colour figure can be viewed at wileyonlinelibrary.com]

Using the same experimental strategy, we evaluated TORC1 activation by monitoring Rps6 phosphorylation. The NA and WA strains showed similar phenotypes, increasing Rps6 phosphorylation after the glutamine pulse (Figure 4a,c). Nevertheless, SA strain seems to have a greater activation, whereas WE strain shows both a lower activation and a decay at $30 \mathrm{~min}$, in concordance with the results obtained for Sch9 phosphorylation (Figure 4). Overall, with exception of the WE strain, all strains showed the expected behaviour, in agreement with previously described observations where Rps6 phosphorylation increased over time in a nitrogen upshift experiment (Gonzalez et al., 2015).

Altogether, the results obtained for Sch9 and Rps6 phosphorylation confirmed the existence of phenotypic differences between strains, showing SA strain as the one that has a greater TORC1 activation and the WE strain as the one with the less sustained one. This general conclusion agrees with the results obtained by our method, although a direct comparison between them is not possible. Nevertheless, we compared these results with the obtained results using the microculture method, focusing in the first $4 \mathrm{~h}$ after the nitrogen pulse, where the first luciferase expression maximum appears (Figure $3 \mathrm{~b}$ and Table 3). When we compared the maximum luminescence and the area under the curve achieved between strains, the results resemble the ones obtained by immunoblot of Rps6, with the SA and WE strains having extreme phenotypes and the NA and WA strains having similar intermediate phenotypes (compare Figure $4 c$ at time 30 min with Figure $3 b)$. Moreover, this novel methodology seems to recapitulate the EGOC-independent activation of TORC1 observed by immunoblot of Sch9, because even though the WE strain exhibited a great activation $5 \mathrm{~min}$ after the glutamine pulse, it lacked a reactivation at $30 \mathrm{~min}$, whereas the SA strain showed the greater activation at this time (compare Figure $4 \mathrm{~b}$ at time 30 min with Figure $3 \mathrm{~b}$ ), consistent with the previously described activation of TORC1 independent of EGOC (Stracka et al., 2014).

In general, our method is based on a transcriptional reporter, making an unfavourable direct comparison with the results obtained by detection of a posttranslational modification by immunoblot. Additionally, the temporary window used in the microculture experiment is greater than that the used for immunoblotting experiments $(4 \mathrm{~h}$ vs $30 \mathrm{~min}$, respectively). Therefore, our method has limitations; for example, it can take more time to overcome the background noise in this type of experiments than in a Western blot; in addition, cells take more time to transcribe and then translate the luciferase protein than to directly phosphorylate Rps6 or Sch9. Thus, this longer temporary window is also consistent with a possible evaluation of EGOC-independent activation of TORC1 by our method, because preferred nitrogen sources (such as glutamine) are capable of sustained TORC1 activation (Gonzalez \& Hall, 2017; Stracka et al., 2014).

We corroborated that out method can detect the EGOC-independent activation of TORC1 by repeating the nitrogen upshift experiments using leucine, an amino acid that activates TORC1 only in a EGOC-dependent (Gtr1-dependent) manner and is incapable of sustained TORC1 activity (Stracka et al., 2014). The results showed that leucine is unable to increase the luminescence signal after a nitrogen upshift in the four strains has been evaluated, supporting the idea that our system only detects EGOC-independent activation of TORC1 by preferred nitrogen sources (Figure 5). We confirmed this result by repeating the experiment in the SA and WE strains (the phenotypically more different strains) carrying GTR1 deletion (gtr1 $\Delta$ ), confirming that this mutation has minimal effects on the TORC1 activation by a

TABLE 3 Parameters associated with luminescence curves

\begin{tabular}{|c|c|c|c|c|c|c|}
\hline Strain & $\operatorname{Max}_{0-12} \pm S D$ (a.u.) & Time $_{0-12} \pm S D(h)$ & $\mathrm{AUC}_{0-12} \pm S D$ (a.u.) & $\mathrm{Max}_{0-4} \pm S D$ (a.u.) & Time $_{0-4} \pm S D(h)$ & $\mathrm{AUC}_{0-4} \pm S D$ (a.u.) \\
\hline NA & $309 \pm 50$ & $5.2 \pm 0.6$ & $1,230 \pm 176$ & $234 \pm 44$ & $2.4 \pm 0.6$ & $494 \pm 40$ \\
\hline SA & $410 \pm 7$ & $1.7 \pm 0.3$ & $1,324 \pm 113$ & $410 \pm 7$ & $1.7 \pm 0.3$ & $930 \pm 66$ \\
\hline WA & $296 \pm 43$ & $3.5 \pm 2.9$ & $1,408 \pm 313$ & $257 \pm 13$ & $2.3 \pm 0.9$ & $622 \pm 44$ \\
\hline WE & $289 \pm 14$ & $8.4 \pm 0.3$ & $1,213 \pm 126$ & $121 \pm 8$ & $2.3 \pm 0.5$ & $273 \pm 36$ \\
\hline
\end{tabular}

Note. Max: maximum luminescence; Time: maximum luminescence time; AUC: area under the curve of luminescence; 0-12: 0-12 hours' interval; 0-4: 0-4 hours' interval; SD: standard deviation; NA: North American; SA: Sake; WA: West African; WE: Wine/European. 
(a)
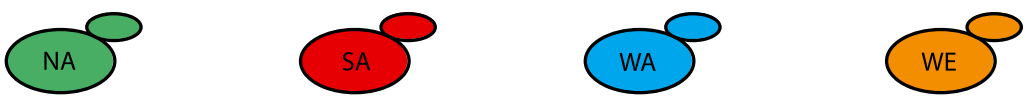

$\begin{array}{cllll}\begin{array}{c}\text { Time (min) } \\ \text { pRps6 }\end{array} & 0 & 5 & 15 & 30 \\ & & & & \end{array}$

$\begin{array}{llll}0 & 5 & 15 & 30\end{array}$

0

515

30

$\begin{array}{llll}0 & 5 & 15 & 30\end{array}$

Total Rps6

Sch9-3xHA
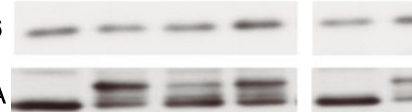

(b)

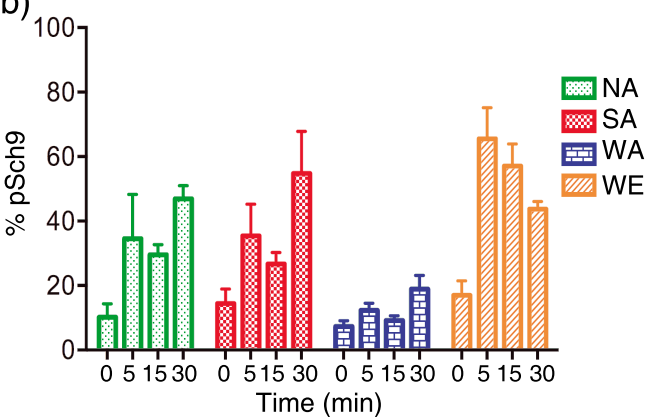

(C)

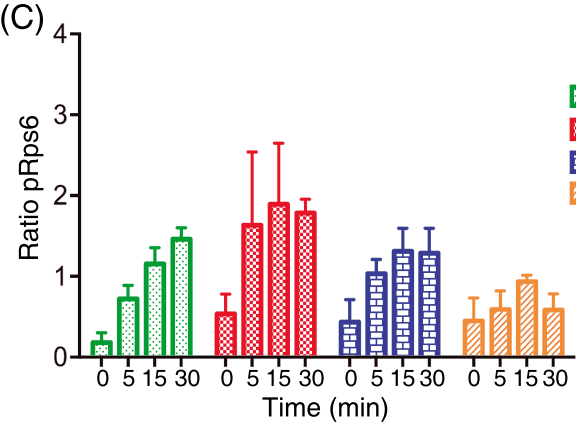

FIGURE 4 Measuring TORC1 activity by immunoblot. (a) Western blot for the strains NA, SA, WA, and WE. In the case of Rps6, phosphorylated (pRps6) and total Rps6 protein levels were evaluated using specific antibodies. In the Sch9 phosphorylation, an antibody that recognizes the 3xHA epitope was used, which gives an upper band and a lower band that are representative of phosphorylated (pSch9) and nonphosphorylated (nonpSch9) isoforms, respectively. Time 0 min corresponds to the addition of glutamine. (b) Quantification of Sch9 phosphorylation. The "\% pSch9" is the ratio between the pSch9 band intensity and the sum of the pSch9 and non-pSch9 band intensities, multiplied by 100. Plotted values correspond to the average of three biological replicates, with their standard error represented by bars (mean \pm SEM). (c) Quantification of Rps6 phosphorylation. The "Ratio pRps6" is the ratio between pRps6 band intensity and the total Rps6 band intensity, normalized by the mean value of all the ratios obtained. Plotted values correspond to the average of three biological replicates, with their standard error represented by bars (mean \pm SEM). NA: North American; SA: Sake; WA: West African; WE: Wine/European [Colour figure can be viewed at wileyonlinelibrary.com]

(a)
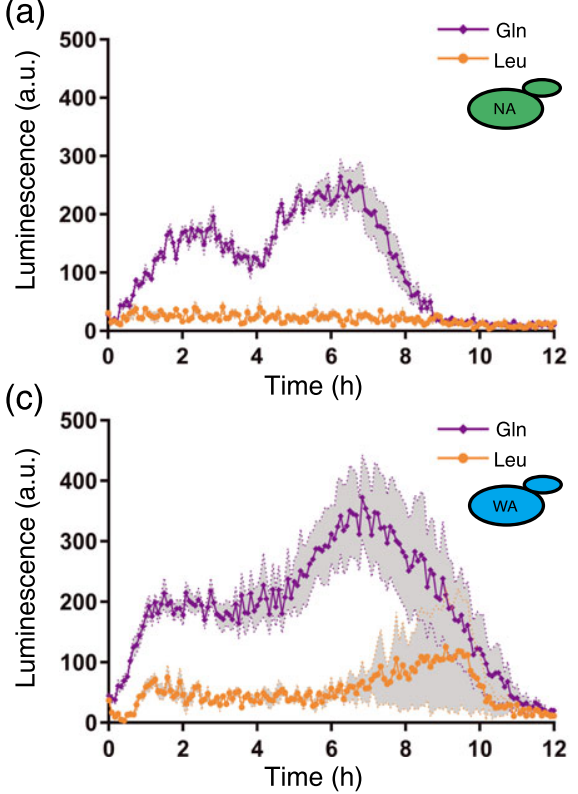

(b)
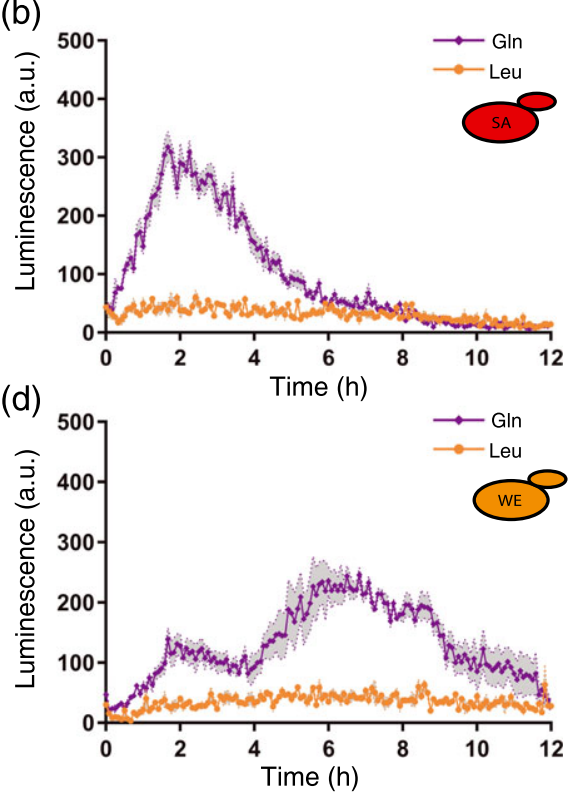

FIGURE 5 Nitrogen upshift experiment using glutamine and leucine. Luminescence (Lum) differences between strains (a) NA, (b) SA, (c) WA, and (d) WE were evaluated in microculture conditions after a pulse of glutamine (Gln) and a pulse of leucine (Leu). The luminescence was recorded until $12 \mathrm{~h}$ after nitrogen pulse. Time $\mathrm{O} \mathrm{h}$ corresponds to the addition of glutamine or leucine. Plotted values correspond to the average of three biological replicates, with their standard error represented by shadow regions (mean \pm SEM). NA: North American; SA: Sake; WA: West African; WE: Wine/European [Colour figure can be viewed at wileyonlinelibrary.com]

preferred nitrogen source such as glutamine (Figure 6a,c). Conversely, when we used leucine, a nonpreferred nitrogen source, which activates TORC1 in an EGOC-dependent manner, we did not observe an increase in the reporter gene expression in the WT and gtr1 $\Delta$ strains
(Figure 6b,d). Altogether, these results are consistent with the idea that our system is capable to indirectly detect the EGOC-independent activation of TORC1, which occurs only by preferred nitrogen sources such as glutamine. 
(a)
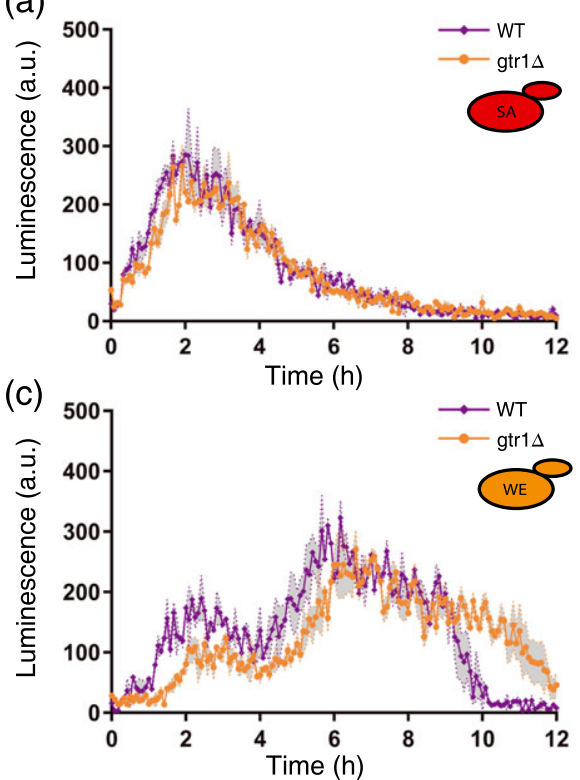

(b)
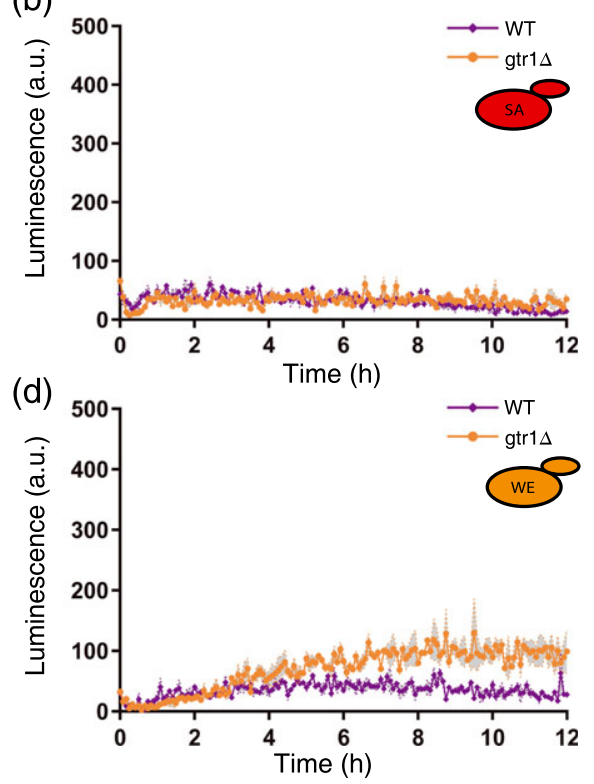

FIGURE 6 Nitrogen upshift experiment using gtr1 $\triangle$ strains. Luminescence (Lum) differences between strains SA (a and b) and WE (c and d) were evaluated in microculture conditions after a pulse of glutamine ( $a$ and $c$ ) or leucine ( $b$ and $d$ ). The luminescence was recorded until $12 \mathrm{~h}$ after nitrogen pulse. Time $\mathrm{O} \mathrm{h}$ corresponds to the addition of glutamine or leucine. Plotted values correspond to the average of three biological replicates, with their standard error represented by shadow regions (mean \pm SEM). NA: North American; SA: Sake; WA: West African; WE: Wine/ European [Colour figure can be viewed at wileyonlinelibrary.com]

In general, the destabilized version of the firefly luciferase reporter gene has become an ideal tool to assess gene expression dynamics in living cells, allowing to measure the transcriptional activity of genes regulated by nutrient availability, osmotic stress, and oxidative stress in yeasts (Dolz-Edo et al., 2013; Rienzo et al., 2015). In this sense, we used the luciferase reporter gene to record the transcriptional activity of RPL26A gene-which is a gene known to be expressed upon TORC1 activation-using nitrogen upshift experiments, and the results showed strong activation RPL26A only by a preferred nitrogen source (glutamine).

In conclusion, we report a new method based on growth under microculture conditions and using the luciferase reporter gene for indirect measuring of TORC1 EGOC-independent activity, with its results being in partial agreement with those obtained through traditional methodologies based on the relative estimation of the phosphorylation of Sch9 and Rps6 by immunoblot. The results obtained indicate that there are phenotypic differences in the kinetics of TORC1 activation by glutamine between distinct strains representative of $S$. cerevisiae clean lineages, with greater differences between Y12 (SA) and DBVPG6765 (WE) strains. This opens the possibility to use this new methodology to investigate the molecular basis of TORC1 activation by different nitrogen sources using high throughput approaches, such as BSA, linkage analysis, or comparative genomics, which require phenotyping of numerous strains.

\section{ACKNOWLEDGEMENTS}

We thank Robbie Loewith (University of Geneva, Switzerland) for pJU733 plasmid, and Walter Tapia, Marco Gaete, Camila Bastías, and Wolfgang Oppliger for technical help. This work was supported by CONICYT/FONDEQUIP (Grant EQM130158), CONICYT/FONDECYT
(Grant 1150522) to C. M., CONICYT/FONDECYT (Grant 11170158) and CONICYT/PCI (Grant REDI170239) to F. S., CONICYT/Beca Doctorado Nacional (Grant 21150700) to E. I. K.-P., Instituto Milenio iBio - Iniciativa Científica Milenio-MINECON to L. F. L., and CSIC/iLINK+ (Grant 0946) to J. M. G.

\section{CONFLICT OF INTEREST}

The authors declare no conflict of interest.

\section{ORCID}

Eduardo I. Kessi-Pérez (D) http://orcid.org/0000-0003-4225-9486

Claudio Martínez (D) http://orcid.org/0000-0001-8564-9287

\section{REFERENCES}

Binda, M., Peli-Gulli, M. P., Bonfils, G., Panchaud, N., Urban, J., Sturgill, T. W., ... De Virgilio, C. (2009). The Vam6 GEF controls TORC1 by activating the EGO complex. Molecular Cell, 35(5), 563-573. https://doi. org/10.1016/j.molcel.2009.06.033

Bonfils, G., Jaquenoud, M., Bontron, S., Ostrowicz, C., Ungermann, C., \& De Virgilio, C. (2012). Leucyl-tRNA synthetase controls TORC1 via the EGO complex. Molecular Cell, 46(1), 105-110. https://doi.org/ 10.1016/j.molcel.2012.02.009

Brice, C., Sanchez, I., Bigey, F., Legras, J. L., \& Blondin, B. (2014). A genetic approach of wine yeast fermentation capacity in nitrogen-starvation reveals the key role of nitrogen signaling. BMC Genomics, 15, 495. https://doi.org/10.1186/1471-2164-15-495

Broach, J. R. (2012). Nutritional control of growth and development in yeast. Genetics, 192(1), 73-105. https://doi.org/10.1534/ genetics.111.135731

Chantranupong, L., Wolfson, R. L., \& Sabatini, D. M. (2015). Nutrient-sensing mechanisms across evolution. Cell, 161(1), 67-83. https://doi.org/ 10.1016/j.cell.2015.02.041 
Conrad, M., Schothorst, J., Kankipati, H. N., Van Zeebroeck, G., RubioTexeira, M., \& Thevelein, J. M. (2014). Nutrient sensing and signaling in the yeast Saccharomyces cerevisiae. FEMS Microbiology Reviews, 38(2), 254-299. https://doi.org/10.1111/1574-6976.12065

Contreras, A., Garcia, V., Salinas, F., Urzua, U., Ganga, M. A., \& Martinez, C. (2012). Identification of genes related to nitrogen uptake in wine strains of Saccharomyces cerevisiae. World Journal of Microbiology and Biotechnology, 28(3), 1107-1113. https://doi.org/10.1007/s11274011-0911-3

Crepin, L., Nidelet, T., Sanchez, I., Dequin, S., \& Camarasa, C. (2012). Sequential use of nitrogen compounds by Saccharomyces cerevisiae during wine fermentation: A model based on kinetic and regulation characteristics of nitrogen permeases. Applied and Environmental Microbiology, 78(22), 8102-8111. https://doi.org/10.1128/AEM.02294-12

Cubillos, F. A., Brice, C., Molinet, J., Tisne, S., Abarca, V., Tapia, S. M., ... Martinez, C. (2017). Identification of nitrogen consumption genetic variants in yeast through QTL mapping and bulk segregant RNA-Seq analyses. G3 (Bethesda), 7(6), 1693-1705. https://doi.org/10.1534/ g3.117.042127

Cubillos, F. A., Louis, E. J., \& Liti, G. (2009). Generation of a large set of genetically tractable haploid and diploid Saccharomyces strains. FEMS Yeast Research, 9(8), 1217-1225. https://doi.org/10.1111/j.15671364.2009.00583.x

Dolz-Edo, L., Rienzo, A., Poveda-Huertes, D., Pascual-Ahuir, A., \& Proft, M. (2013). Deciphering dynamic dose responses of natural promoters and single cis elements upon osmotic and oxidative stress in yeast. Molecular and Cellular Biology, 33(11), 2228-2240. https://doi.org/10.1128/ MCB.00240-13

Gietz, R. D., \& Schiestl, R. H. (2007). Large-scale high-efficiency yeast transformation using the LiAc/SS carrier DNA/PEG method. Nature Protocols, 2(1), 38-41. https://doi.org/10.1038/nprot.2007.15

Gonzalez, A., \& Hall, M. N. (2017). Nutrient sensing and TOR signaling in yeast and mammals. EMBO J, 36(4), 397-408. https://doi.org/ 10.15252/embj.201696010

Gonzalez, A., Shimobayashi, M., Eisenberg, T., Merle, D. A., Pendl, T., Hall, M. N., \& Moustafa, T. (2015). TORC1 promotes phosphorylation of ribosomal protein S6 via the AGC kinase Ypk3 in Saccharomyces cerevisiae. PLoS One, 10(3), e0120250. https://doi.org/10.1371/journal.pone. 0120250

Gutierrez, A., Beltran, G., Warringer, J., \& Guillamon, J. M. (2013). Genetic basis of variations in nitrogen source utilization in four wine commercial yeast strains. PLoS One, 8(6), e67166. https://doi.org/10.1371/ journal.pone.0067166

Hatakeyama, R., \& De Virgilio, C. (2016). Unsolved mysteries of Rag GTPase signaling in yeast. Small GTPases, 7(4), 239-246. https://doi. org/10.1080/21541248.2016.1211070

Ibstedt, S., Stenberg, S., Bages, S., Gjuvsland, A. B., Salinas, F., Kourtchenko, O., ... Warringer, J. (2015). Concerted evolution of life stage performances signals recent selection on yeast nitrogen use. Molecular Biology and Evolution, 32(1), 153-161. https://doi.org/10.1093/ molbev/msu285

Jara, M., Cubillos, F. A., Garcia, V., Salinas, F., Aguilera, O., Liti, G., \& Martinez, C. (2014). Mapping genetic variants underlying differences in the central nitrogen metabolism in fermenter yeasts. PLoS One, 9(1), e86533. https://doi.org/10.1371/journal.pone.0086533

Kessi-Perez, E. I., Araos, S., Garcia, V., Salinas, F., Abarca, V., Larrondo, L. F., ... Cubillos, F. A. (2016). RIM15 antagonistic pleiotropy is responsible for differences in fermentation and stress response kinetics in budding yeast. FEMS Yeast Research, 16(3). https://doi.org/10.1093/femsyr/ fow021, fow021

Kim, A., \& Cunningham, K. W. (2015). A LAPF/phafin1-like protein regulates TORC1 and lysosomal membrane permeabilization in response to endoplasmic reticulum membrane stress. Molecular Biology of the Cell, 26(25), 4631-4645. https://doi.org/10.1091/mbc.E15-08-0581
Liti, G., Carter, D. M., Moses, A. M., Warringer, J., Parts, L., James, S. A., ... Louis, E. J. (2009). Population genomics of domestic and wild yeasts. Nature, 458(7236), 337-341. https://doi.org/10.1038/nature07743

Loewith, R., \& Hall, M. N. (2011). Target of rapamycin (TOR) in nutrient signaling and growth control. Genetics, 189(4), 1177-1201. https://doi. org/10.1534/genetics.111.133363

Loewith, R., Jacinto, E., Wullschleger, S., Lorberg, A., Crespo, J. L., Bonenfant, D., ... Hall, M. N. (2002). Two TOR complexes, only one of which is rapamycin sensitive, have distinct roles in cell growth control. Molecular Cell, 10(3), 457-468.

Mackay, T. F., Stone, E. A., \& Ayroles, J. F. (2009). The genetics of quantitative traits: Challenges and prospects. Nature Reviews. Genetics, 10(8), 565-577. https://doi.org/10.1038/nrg2612

Michel, A. H., Hatakeyama, R., Kimmig, P., Arter, M., Peter, M., Matos, J., ... Kornmann, B. (2017). Functional mapping of yeast genomes by saturated transposition. Elife, 6. https://doi.org/10.7554/eLife.23570

Oliveira, A. P., Dimopoulos, S., Busetto, A. G., Christen, S., Dechant, R., Falter, L., ... Stelling, J. (2015). Inferring causal metabolic signals that regulate the dynamic TORC1-dependent transcriptome. Mol Syst Biol, 11(4), 802. https://doi.org/10.15252/msb.20145475

Powis, K., \& De Virgilio, C. (2016). Conserved regulators of Rag GTPases orchestrate amino acid-dependent TORC1 signaling. Cell Discov, 2, 15049. https://doi.org/10.1038/celldisc.2015.49

Pretorius, I. S. (2000). Tailoring wine yeast for the new millennium: Novel approaches to the ancient art of winemaking. Yeast, 16(8), 675-729. https://doi.org/10.1002/1097-0061(20000615)16:8<675::AIDYEA585>3.0.CO;2-B

R-Core-Team (2013). R: A language and environment for statistical computing . Vienna, Austria: R Foundation for Statistical Computing. Retrieved from http://www.r-project.org/

Rienzo, A., Pascual-Ahuir, A., \& Proft, M. (2012). The use of a real-time luciferase assay to quantify gene expression dynamics in the living yeast cell. Yeast, 29(6), 219-231. https://doi.org/10.1002/yea.2905

Rienzo, A., Poveda-Huertes, D., Aydin, S., Buchler, N. E., Pascual-Ahuir, A., \& Proft, M. (2015). Different mechanisms confer gradual control and memory at nutrient- and stress-regulated genes in yeast. Molecular and Cellular Biology, 35(21), 3669-3683. https://doi.org/10.1128/ MCB.00729-15

Salinas, F., Cubillos, F. A., Soto, D., Garcia, V., Bergstrom, A., Warringer, J., ... Martinez, C. (2012). The genetic basis of natural variation in oenological traits in Saccharomyces cerevisiae. PLoS One, 7(11), e49640. https:// doi.org/10.1371/journal.pone.0049640

Salinas, F., de Boer, C. G., Abarca, V., Garcia, V., Cuevas, M., Araos, S., ... Cubillos, F. A. (2016). Natural variation in non-coding regions underlying phenotypic diversity in budding yeast. Scientific Reports, 6, 21849. https://doi.org/10.1038/srep21849

Stracka, D., Jozefczuk, S., Rudroff, F., Sauer, U., \& Hall, M. N. (2014). Nitrogen source activates TOR (target of rapamycin) complex 1 via glutamine and independently of Gtr/Rag proteins. The Journal of Biological Chemistry, 289(36), 25010-25020. https://doi.org/10.1074/jbc. M114.574335

Taillandier, P., Ramon Portugal, F., Fuster, A., \& Strehaiano, P. (2007). Effect of ammonium concentration on alcoholic fermentation kinetics by wine yeasts for high sugar content. Food Microbiology, 24(1), 95-100. https://doi.org/10.1016/j.fm.2006.04.002

Tanigawa, M., \& Maeda, T. (2017). An in vitro TORC1 kinase assay that recapitulates the Gtr-independent glutamine-responsive TORC1 activation mechanism on yeast vacuoles. Molecular and Cellular Biology, 37(14). https://doi.org/10.1128/MCB.00075-17

Tesniere, C., Brice, C., \& Blondin, B. (2015). Responses of Saccharomyces cerevisiae to nitrogen starvation in wine alcoholic fermentation. Applied Microbiology and Biotechnology, 99(17), 7025-7034. https://doi.org/ 10.1007/s00253-015-6810-z

Ukai, H., Araki, Y., Kira, S., Oikawa, Y., May, A. I., \& Noda, T. (2018). Gtr/ Ego-independent TORC1 activation is achieved through a glutamine- 
sensitive interaction with Pib2 on the vacuolar membrane. PLoS Genet ics, 14(4), e1007334. https://doi.org/10.1371/journal.pgen.1007334

Urban, J., Soulard, A., Huber, A., Lippman, S., Mukhopadhyay, D., Deloche, O., ... Loewith, R. (2007). Sch9 is a major target of TORC1 in Saccharomyces cerevisiae. Molecular Cell, 26(5), 663-674. https://doi.org/ 10.1016/j.molcel.2007.04.020

Varela, C., Pizarro, F., \& Agosin, E. (2004). Biomass content governs fermentation rate in nitrogen-deficient wine musts. Applied and Environmental Microbiology, 70(6), 3392-3400. https://doi.org/10.1128/ AEM.70.6.3392-3400.2004

Varlakhanova, N. V., Mihalevic, M. J., Bernstein, K. A., \& Ford, M. G. J. (2017). Pib2 and the EGO complex are both required for activation of TORC1. Journal of Cell Science, 130(22), 3878-3890. https://doi.org/ $10.1242 /$ jcs. 207910

Warringer, J., Zorgo, E., Cubillos, F. A., Zia, A., Gjuvsland, A., Simpson, J. T., ... Blomberg, A. (2011). Trait variation in yeast is defined by population history. PLoS Genetics, 7(6), e1002111. https://doi.org/10.1371/journal.pgen.1002111

Yerlikaya, S., Meusburger, M., Kumari, R., Huber, A., Anrather, D., Costanzo, M., ... Loewith, R. (2016). TORC1 and TORC2 work together to regulate ribosomal protein S6 phosphorylation in Saccharomyces cerevisiae. Molecular Biology of the Cell, 27(2), 397-409. https://doi. org/10.1091/mbc.E15-08-0594

Yin, X., Goudriaan, J., Lantinga, E. A., Vos, J., \& Spiertz, H. J. (2003). A flexible sigmoid function of determinate growth. Annals of Botany, 91(3), 361-371.

\section{SUPPORTING INFORMATION}

Additional supporting information may be found online in the Supporting Information section at the end of the article.

How to cite this article: Kessi-Pérez El, Salinas F, Molinet J, et al. Indirect monitoring of TORC1 signalling pathway reveals molecular diversity among different yeast strains. Yeast. 2019;36:65-74. https://doi.org/10.1002/yea.3351 